تأثير ميل القناة على خصائص الجريان للمسقط المائي العمودي والمنحرف

احمد يونس محمد

مدرس مساعد - جامعة الموصل/كلية الهندسة/قسم الموارد المائية

\title{
الخلاصة
}

يقدم هذا البحث دراسة عملية وتحليل لتأثير ميل القناة على المسقط المائي فضلا عن مقارنة بين المسقط العمــودي و المنحرف في قناة مستطيلة مختلفة الميول.

تم إيجاد علاقات بين عمق المئية الماء الحرج و وعمق الماء فوق حافة المسقط المائي العمودي و المنحرف ولجميع الميول,

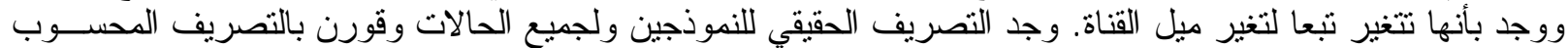

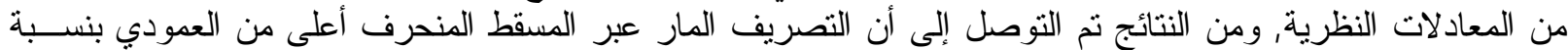

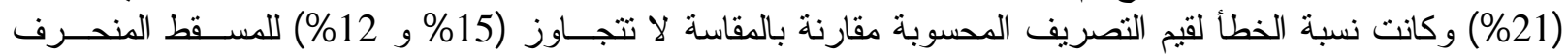

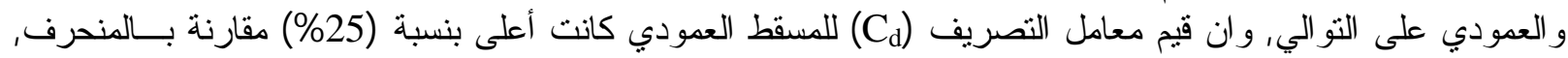
كما تم التوصل إلى علاقات بين ميل القتاة ونسبة عمق الماء فوق حافة المسقط إلى عمق الماء الحــرج (h/he

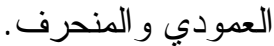
الكلمات الادلة : المسقط المائي, الهدار, العمق الحرج, العمق عند حافة المسقط, ميل القناة

\section{Effecting of Channel Slope on Flow Characteristics for Straight Vertical and Skew Free overfall}

\author{
Ahmed Y.Mohammed \\ Asst. Lect.- Univ. of Mosul, Coll. of Eng., Dept. of Water Resources, Eng.
}

\begin{abstract}
This paper presents an experimental study and analysis for effect of channel slope on straight vertical and skew free overfall for a rectangular channel with different slopes. The relationship between the critical depth and brink depth for both vertical and skew free overfall are found and shown to be influenced by channel slope. The measured discharge from standard weir was compared with calculated discharge from theoretical equation and found that the discharge over skewed model is greater by $(21 \%)$ from straight vertical, and the error percentage for calculated and measured discharge not exceed (15\% and 12\%) for skewed and vertical models respectively for all slopes. The discharge coefficient for vertical model is greater by $(25 \%)$ than skew, relationships between end and critical depths $\left(h_{e} / h_{c}\right)$ with channel slope for straight vertical and skew free overfall were calculated.
\end{abstract}




\begin{tabular}{|c|c|c|}
\hline 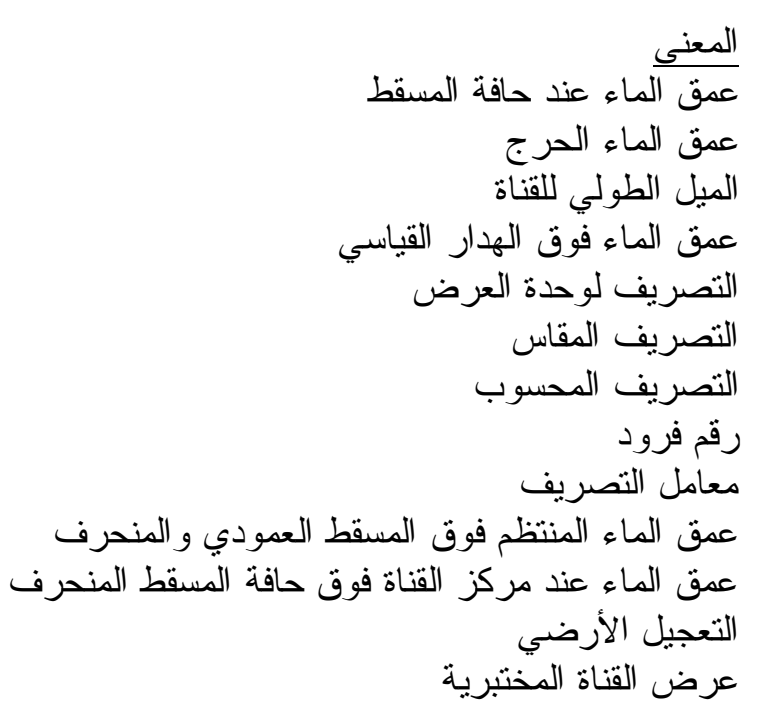 & $\begin{array}{l}\frac{\text { الوحدة }}{\mathrm{L}} \\
\mathrm{L} \\
- \\
\mathrm{L} \\
\mathrm{L}^{3} / \mathrm{T} . \mathrm{L} \\
\mathrm{L}^{3} / \mathrm{T} \\
\mathrm{L}^{3} / \mathrm{T} \\
- \\
- \\
\mathrm{L} \\
\mathrm{L} \\
\mathrm{L} / \mathrm{T}^{2} \\
\mathrm{~L}\end{array}$ & $\begin{array}{c}\frac{j \mathrm{j}}{\mathrm{h}_{\mathrm{e}}} \\
\mathrm{h}_{\mathrm{c}} \\
\mathrm{S}_{\mathrm{o}} \\
\mathrm{H}_{\mathrm{w}} \\
\mathrm{q} \\
\mathrm{Q}_{\mathrm{m}} \\
\mathrm{Q}_{\mathrm{cal}} \\
\mathrm{F}_{\mathrm{o}} \\
\mathrm{C}_{\mathrm{d}} \\
\mathrm{h}_{\mathrm{o}} \\
\mathrm{h}_{\mathrm{e}} \mathrm{center} \\
\mathrm{g} \\
\mathrm{b}\end{array}$ \\
\hline
\end{tabular}

المقدمة:

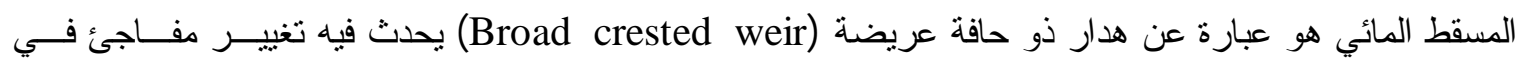

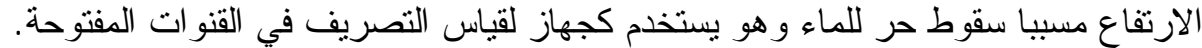

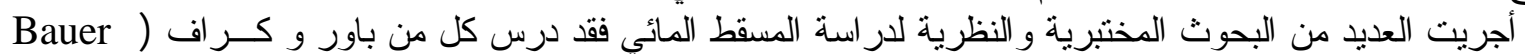
هائة (1971) (and Graf

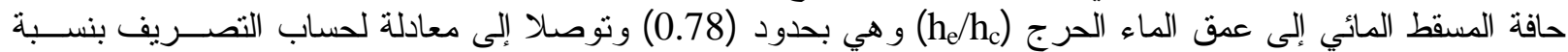

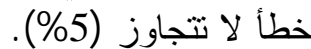

أما راجارتتام وآخرون (Rajaratnam et. al.) (1976) وديفس و آخرون (Davis et. al) (1998) فقد درســا

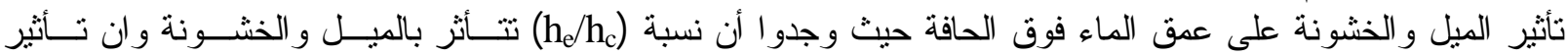
الخشونة يزداد كلما زاد الميل. تلئ. مارجي (Marchi) (1993) وفيرو (1999) (1999) درسا المسقط المائي نظريا في قناة مسـنطيلة باســتخدام

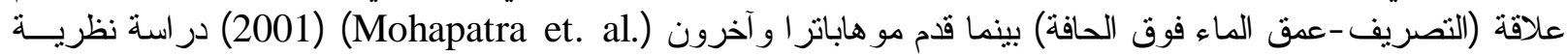

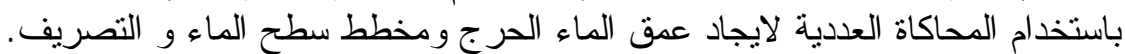

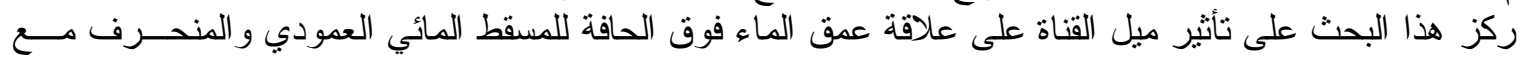
عمق الماء الحرج (h/he). كما نم إيجاد علاقة نظرية لإيجاد النصريف المار فوق المسقط المائي لكل حالة من حسالات الميل ومقارنتها بالتصريف الحقيقي المار بالقناة. فضلا عن إيجاد علاقات نظرية لكل من حالات الميل مع نســبة (he/he للمسقط العمودي و المنحرف.

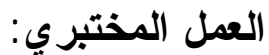

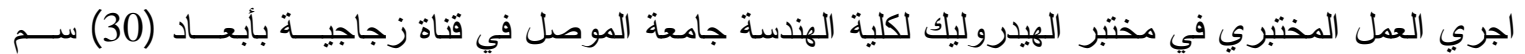

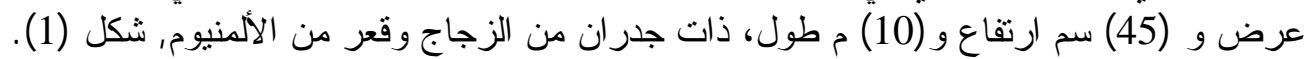

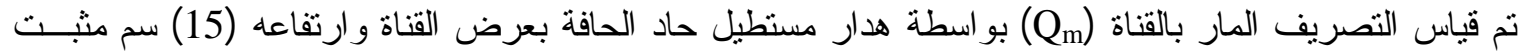
في نهاية القناة ويرفع بعد كل عملية قياس للتصريف لضمان الفران عدم تكون ماء ذيلي مؤخر النماذج. و ان معادلة التصريف لهذا الهذار هي (الطائي, 2002 ).

$\mathrm{Q}_{\mathrm{m}}=0.64 * \mathrm{H}_{\mathrm{w}}^{3 / 2}$

حيث ان (Qm) التصريف المقاس (1/s)

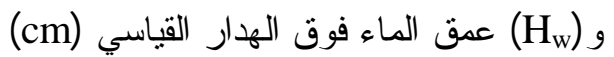

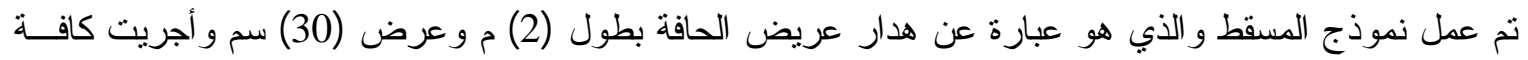
القياسات مؤخر هذا الهدار حيث أخذت حافة شاقولية ومنحرفة بز اوية (30) درجة عن جدار القنــاة, الأشـــال (2 و 3). 


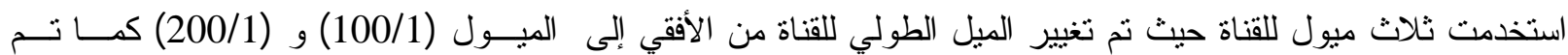

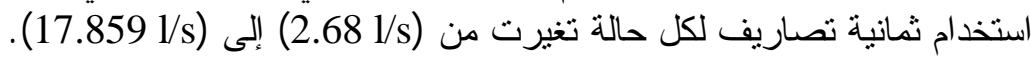

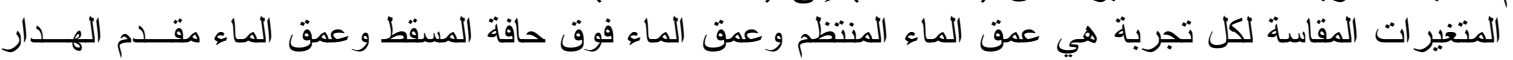
القياسي على التو الي التصريف لوحدة العرض (q) حسب من المعادلة:

$\mathrm{q}=\mathrm{Q}_{\mathrm{m}} / \mathrm{b}$

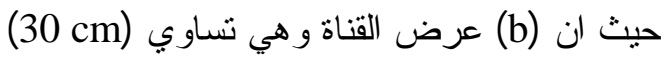
أما رقم فرود (Fo ) فحسب من المعادلة:

$\mathrm{F}_{\mathrm{o}}=\mathrm{q} / \mathrm{h}_{\mathrm{o}} \sqrt{g h_{o}}$

حيث ان (g) هو التعجيل الارضي (m/s²) في حين وجد العمق الحرج (h) من الأبل المعادلة:

$\mathrm{h}_{\mathrm{c}}=\sqrt[3]{q^{2} / g}$

$\mathrm{Q}_{\mathrm{cal}}=\mathrm{b} \sqrt{g h_{e}^{3}}$

و التصريف المحسوب فوجد من المعادلة

$$
\mathrm{Q}_{\mathrm{cal}}=\mathrm{b} \sqrt{g h_{e}^{3}}
$$

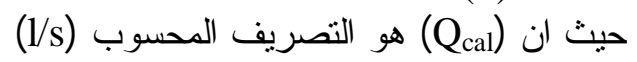

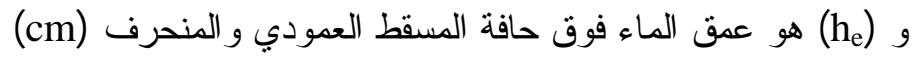

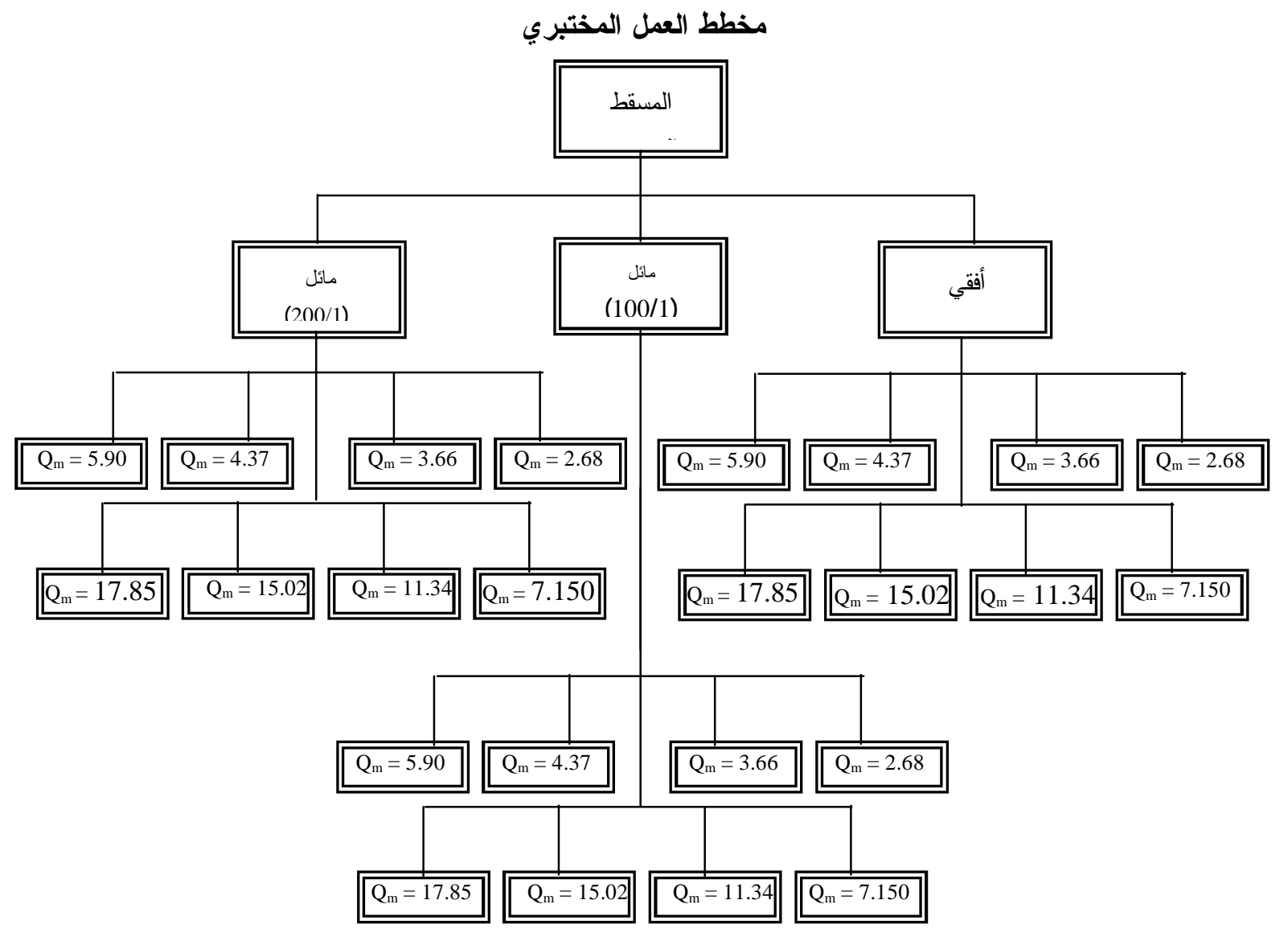




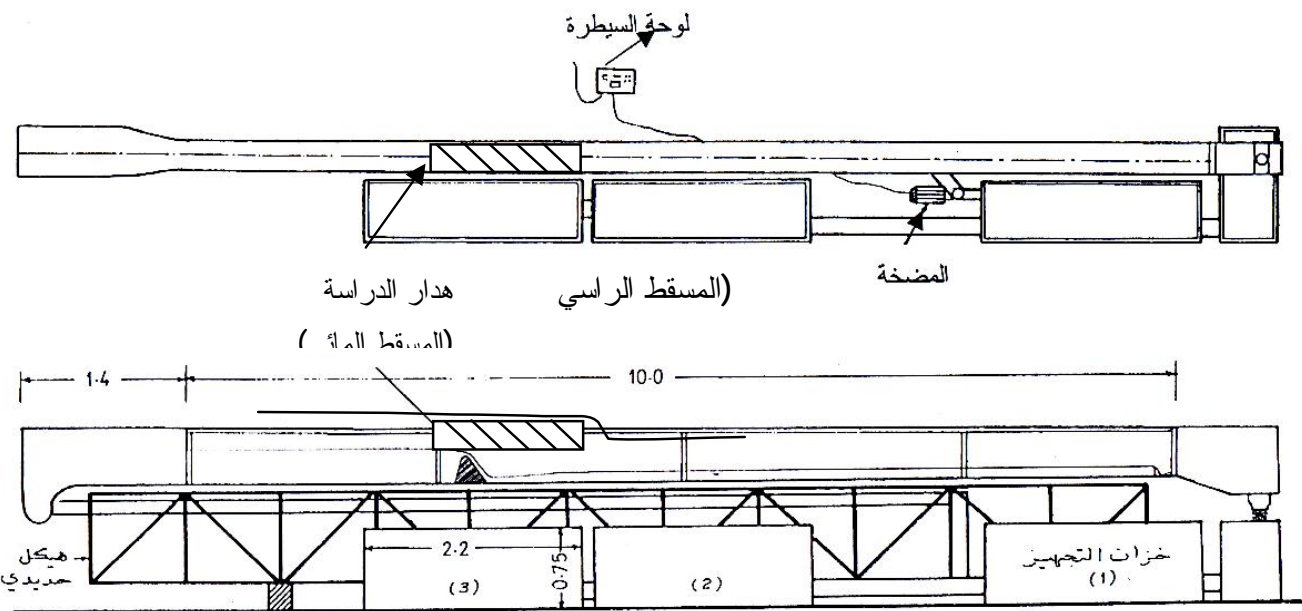

पाषासा

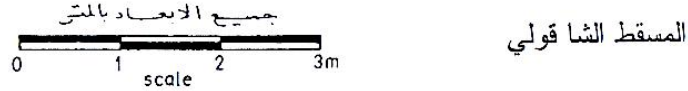

الثكل (1) القناة المختبرية

Flow

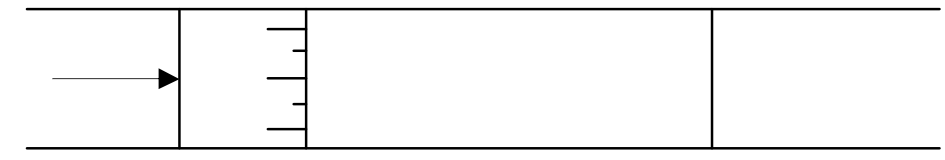

(a)

Flow

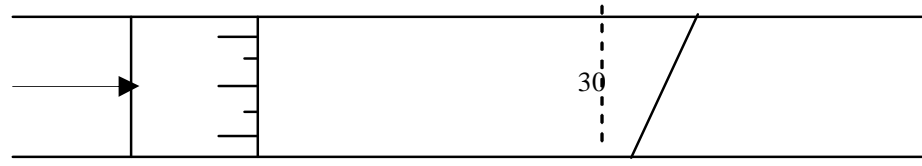

(b)

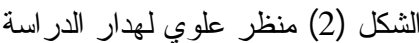

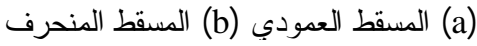

Flow

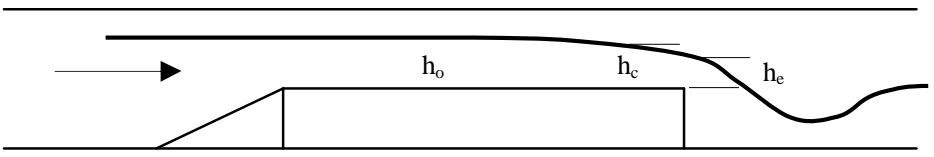

(a)

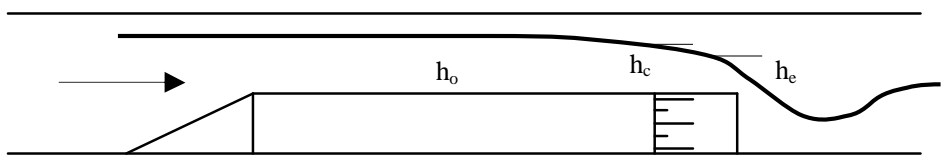

(b)

الثكل (3) منظر جانبي لهدار الدر اسة

(a) المسقط العمودي (b) المسقط المنحرف (3) (a) 


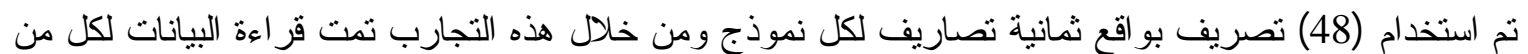

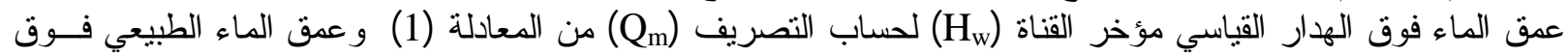

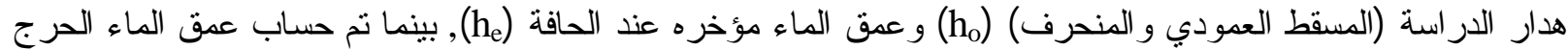

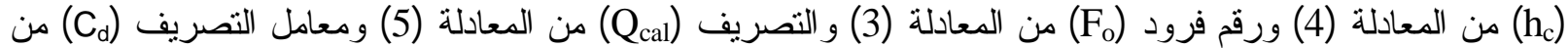

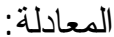

$\mathrm{C}_{\mathrm{d}}=\mathrm{Q}_{\mathrm{m}} / \mathrm{Q}_{\mathrm{cal}}$

وجميع هذه البيانات موضحة بالجداول (1 -6).

الجدول (1) البيانات المختبرية والمحسوبة للمسقط العودي الأفقي

\begin{tabular}{|c|c|c|c|c|c|c|c|c|c||c|}
\hline $\begin{array}{c}\mathrm{H}_{\mathrm{w}} \\
(\mathrm{cm})\end{array}$ & $\begin{array}{c}\mathrm{Q}_{\mathrm{m}} \\
(\mathrm{l} / \mathrm{s})\end{array}$ & $\begin{array}{c}\mathrm{q} \\
\left(\mathrm{m}^{3} / \mathrm{s} . \mathrm{m}\right)\end{array}$ & $\begin{array}{c}\mathrm{h}_{\mathrm{o}} \\
(\mathrm{cm})\end{array}$ & $\begin{array}{c}\mathrm{h}_{\mathrm{c}} \\
(\mathrm{cm})\end{array}$ & $\begin{array}{c}\mathrm{h}_{\mathrm{e}} \\
(\mathrm{c})\end{array}$ & $\mathrm{F}_{\mathrm{o}}$ & $\mathrm{h}_{\mathrm{e}} / \mathrm{h}_{\mathrm{c}}$ & $\begin{array}{c}\mathrm{Q}_{\mathrm{cal}} \\
(\mathrm{l} / \mathrm{s})\end{array}$ & $\mathrm{C}_{\mathrm{d}}$ & $\begin{array}{c}\text { Sur. Or diff. } \\
\text { of }\left(\mathrm{Q}_{\mathrm{cal}}\right)\end{array}$ \\
\hline \hline 9.2 & 17.859 & 0.060 & 8.2 & 7.122 & 5 & 0.809 & 0.702 & 18.682 & 0.956 & 1.103 \\
\hline 8.2 & 15.028 & 0.050 & 8 & 6.348 & 4.5 & 0.707 & 0.709 & 15.951 & 0.942 & 1.165 \\
\hline \hline 6.8 & 11.349 & 0.038 & 6.8 & 5.264 & 3.7 & 0.681 & 0.703 & 11.893 & 0.954 & 1.189 \\
\hline \hline 5 & 7.155 & 0.024 & 5.2 & 3.871 & 2.6 & 0.642 & 0.672 & 7.005 & 1.021 & 1.182 \\
\hline 4.4 & 5.907 & 0.020 & 4.3 & 3.406 & 2.4 & 0.705 & 0.705 & 6.212 & 0.951 & 0.983 \\
\hline 3.6 & 4.372 & 0.015 & 4 & 2.787 & 1.9 & 0.582 & 0.682 & 4.376 & 0.999 & 1.094 \\
\hline 3.2 & 3.664 & 0.012 & 3.8 & 2.477 & 1.7 & 0.526 & 0.686 & 3.703 & 0.989 & 0.966 \\
\hline \hline 2.6 & 2.683 & 0.009 & 3.5 & 2.013 & 1.4 & 0.436 & 0.696 & 2.768 & 0.969 & 1.156 \\
\hline
\end{tabular}

الجدول (2) البيانات المختبرية و المحسوبة للمسقط العمودي المائل بنسبة (100/1)

\begin{tabular}{|c|c|c|c|c|c|c|c|c|c|c|}
\hline $\begin{array}{c}\mathrm{H}_{\mathrm{w}} \\
(\mathrm{cm})\end{array}$ & $\begin{array}{c}\mathrm{Q}_{\mathrm{m}} \\
(\mathrm{l} / \mathrm{s})\end{array}$ & $\begin{array}{c}\mathrm{q} \\
\left(\mathrm{m}^{3} / \mathrm{s} . \mathrm{m}\right)\end{array}$ & $\begin{array}{c}\mathrm{h}_{\mathrm{o}} \\
(\mathrm{cm})\end{array}$ & $\begin{array}{c}\mathrm{h}_{\mathrm{c}} \\
(\mathrm{cm})\end{array}$ & $\begin{array}{c}\mathrm{h}_{\mathrm{e}} \\
(\mathrm{cm})\end{array}$ & $\mathrm{F}_{\mathrm{o}}$ & $\mathrm{h}_{\mathrm{e}} / \mathrm{h}_{\mathrm{c}}$ & $\begin{array}{c}\mathrm{Q}_{\mathrm{cal}} \\
(\mathrm{l} / \mathrm{s})\end{array}$ & $\mathrm{C}_{\mathrm{d}}$ & $\begin{array}{c}\text { Sur. Or diff. } \\
\text { of }\left(\mathrm{Q}_{\mathrm{cal}}\right)\end{array}$ \\
\hline \hline 9.2 & 17.859 & 0.060 & 7.5 & 7.122 & 4.4 & 0.925 & 0.617 & 15.422 & 1.158 & 1.162 \\
\hline 8.2 & 15.028 & 0.050 & 7.2 & 6.347 & 4 & 0.827 & 0.630 & 13.36 & 1.124 & 1.197 \\
\hline 6.8 & 11.348 & 0.038 & 4.8 & 5.264 & 3.3 & 1.148 & 0.626 & 10.017 & 1.133 & 1.120 \\
\hline 5 & 7.155 & 0.024 & 4 & 3.870 & 2.5 & 0.951 & 0.645 & 6.605 & 1.083 & 1.067 \\
\hline 4.4 & 5.906 & 0.020 & 3.5 & 3.406 & 2 & 0.960 & 0.587 & 4.726 & 1.249 & 1.013 \\
\hline 3.6 & 4.371 & 0.015 & 3.2 & 2.786 & 1.8 & 0.812 & 0.645 & 4.035 & 1.083 & 0.983 \\
\hline 3.2 & 3.663 & 0.012 & 3 & 2.477 & 1.5 & 0.750 & 0.605 & 3.069 & 1.193 & 0.925 \\
\hline \hline 2.6 & 2.683 & 0.009 & 2.5 & 2.012 & 1 & 0.722 & 0.496 & 1.671 & 1.605 & 1.103 \\
\hline
\end{tabular}

الجدول (3) البيانات المختبرية و المحسوبة للمسقط العمودي المائل بنسبة (200/1)

\begin{tabular}{|c|c|c|c||c||c|c|c|c|c|c|}
\hline $\begin{array}{c}\mathrm{H}_{\mathrm{w}} \\
(\mathrm{cm})\end{array}$ & $\begin{array}{c}\mathrm{Q}_{\mathrm{m}} \\
(\mathrm{l} / \mathrm{s})\end{array}$ & $\begin{array}{c}\mathrm{q} \\
\left(\mathrm{m}^{3} / \mathrm{s} . \mathrm{m}\right)\end{array}$ & $\begin{array}{c}\mathrm{h}_{\mathrm{o}} \\
(\mathrm{cm})\end{array}$ & $\begin{array}{c}\mathrm{h}_{\mathrm{c}} \\
(\mathrm{cm})\end{array}$ & $\begin{array}{c}\mathrm{h}_{\mathrm{e}} \\
(\mathrm{cm})\end{array}$ & $\mathrm{F}_{\mathrm{o}}$ & $\mathrm{h}_{\mathrm{e}} / \mathrm{h}_{\mathrm{c}}$ & $\begin{array}{c}\mathrm{Q}_{\mathrm{cal}} \\
(\mathrm{l} / \mathrm{s})\end{array}$ & $\mathrm{C}_{\mathrm{d}}$ & $\begin{array}{c}\text { Sur. Or diff. } \\
\text { of }\left(\mathrm{Q}_{\mathrm{cal}}\right)\end{array}$ \\
\hline \hline 9.2 & 17.859 & 0.060 & 8 & 7.122 & 4.6 & 0.839 & 0.645 & 16.485 & 1.083 & 1.167 \\
\hline 8.2 & 15.028 & 0.050 & 7.5 & 6.347 & 4.3 & 0.778 & 0.677 & 14.899 & 1.008 & 1.159 \\
\hline 6.8 & 11.348 & 0.038 & 5.2 & 5.264 & 3.5 & 1.018 & 0.664 & 10.941 & 1.037 & 1.129 \\
\hline 5 & 7.155 & 0.024 & 4.4 & 3.871 & 2.5 & 0.825 & 0.645 & 6.605 & 1.083 & 1.216 \\
\hline 4.4 & 5.906 & 0.020 & 3.5 & 3.406 & 2.3 & 0.960 & 0.675 & 5.828 & 1.013 & 0.970 \\
\hline 3.6 & 4.371 & 0.015 & 3.3 & 2.786 & 1.8 & 0.776 & 0.645 & 4.035 & 1.083 & 1.134 \\
\hline 3.2 & 3.663 & 0.012 & 3 & 2.477 & 1.5 & 0.750 & 0.605 & 3.069 & 1.193 & 1.043 \\
\hline 2.6 & 2.683 & 0.009 & 2.6 & 2.012 & 1.3 & 0.681 & 0.645 & 2.476 & 1.083 & 1.146 \\
\hline
\end{tabular}


الجدول (4) البيانات المختبرية و المحسوبة للمسقط المنحرف الأفقي

\begin{tabular}{|c||c||c||c||c|c|c||c||c||c|}
\hline \hline $\begin{array}{c}\mathrm{H}_{\mathrm{W}} \\
(\mathrm{cm})\end{array}$ & $\begin{array}{c}\mathrm{Q}_{\mathrm{m}} \\
(\mathrm{l} / \mathrm{s})\end{array}$ & $\begin{array}{c}\mathrm{q} \\
\left(\mathrm{m}^{3} / \mathrm{s} . \mathrm{m}\right)\end{array}$ & $\begin{array}{c}\mathrm{h}_{\circ} \\
(\mathrm{cm})\end{array}$ & $\begin{array}{c}\mathrm{h}_{\mathrm{c}} \\
(\mathrm{cm})\end{array}$ & $\begin{array}{c}\mathrm{h}_{\mathrm{e}}(\mathrm{cm}) \\
\text { center }\end{array}$ & $\mathrm{F}_{\circ}$ & $\mathrm{h}_{\mathrm{e}} / \mathrm{h}_{\mathrm{c}}$ & $\begin{array}{c}\mathrm{Q}_{\text {cal }} \\
(\mathrm{l} / \mathrm{s})\end{array}$ & $\mathrm{C}_{d}$ \\
\hline \hline 9.2 & 17.859 & 0.060 & 7.2 & 7.122 & 4.4 & 0.983 & 0.631 & 19.930 & 0.866 \\
\hline \hline 8.2 & 15.028 & 0.050 & 7 & 6.347 & 4.2 & 0.863 & 0.661 & 18.587 & 0.808 \\
\hline \hline 6.8 & 11.349 & 0.038 & 6.8 & 5.264 & 3.4 & 0.681 & 0.664 & 13.538 & 0.802 \\
\hline \hline 5.2 & 7.589 & 0.025 & 4.6 & 4.025 & 2.45 & 0.818 & 0.608 & 8.281 & 0.916 \\
\hline \hline 4.4 & 5.906 & 0.020 & 3.7 & 3.406 & 2 & 0.883 & 0.587 & 6.107 & 0.967 \\
\hline \hline 3.7 & 4.554 & 0.015 & 3.3 & 2.864 & 1.7 & 0.808 & 0.593 & 4.786 & 0.951 \\
\hline 3.2 & 3.663 & 0.012 & 2.9 & 2.477 & 1.4 & 0.789 & 0.565 & 3.577 & 1.024 \\
\hline \hline 2.7 & 2.839 & 0.009 & 2.5 & 2.090 & 1.3 & 0.764 & 0.622 & 3.200 & 0.887 \\
\hline \hline
\end{tabular}

الجدول (5) البيانات المختبرية و المحسوبة للمسقط المنحرف المائل بنسبة (100/1)

\begin{tabular}{|c||c||c||c||c||c||c|c|c|c|}
\hline \hline $\begin{array}{c}\mathrm{H}_{\mathrm{W}} \\
(\mathrm{cm})\end{array}$ & $\begin{array}{c}\mathrm{Q}_{\mathrm{m}} \\
(\mathrm{l} / \mathrm{s})\end{array}$ & $\begin{array}{c}\mathrm{q} \\
\left(\mathrm{m}^{3} / \mathrm{s} \cdot \mathrm{m}\right)\end{array}$ & $\begin{array}{c}\mathrm{h}_{\mathrm{o}} \\
(\mathrm{cm})\end{array}$ & $\begin{array}{c}\mathrm{h}_{\mathrm{c}} \\
(\mathrm{cm})\end{array}$ & $\begin{array}{c}\mathrm{h}_{\mathrm{e}}(\mathrm{cm}) \\
\mathrm{cente}\end{array}$ & $\mathrm{F}_{\mathrm{o}}$ & $\mathrm{h}_{\mathrm{e}} / \mathrm{h}_{\mathrm{c}}$ & $\begin{array}{c}\mathrm{Q}_{\mathrm{cal}} \\
(\mathrm{l} / \mathrm{s})\end{array}$ & $\mathrm{C}_{\mathrm{d}}$ \\
\hline \hline 9.2 & 17.859 & 0.060 & 6.8 & 7.122 & 4.1 & 1.071 & 0.575 & 17.927 & 0.996 \\
\hline 8.2 & 15.028 & 0.050 & 6.4 & 6.347 & 3.8 & 0.987 & 0.598 & 15.996 & 0.939 \\
\hline \hline 6.8 & 11.349 & 0.038 & 4.1 & 5.264 & 3 & 1.454 & 0.569 & 11.221 & 1.011 \\
\hline 5.2 & 7.589 & 0.025 & 3.6 & 4.025 & 2.2 & 1.182 & 0.546 & 7.046 & 1.077 \\
\hline \hline 4.3 & 5.706 & 0.020 & 2.7 & 3.328 & 1.7 & 1.368 & 0.510 & 4.786 & 1.192 \\
\hline \hline 3.7 & 4.554 & 0.015 & 2.2 & 2.864 & 1.5 & 1.485 & 0.523 & 3.967 & 1.148 \\
\hline 3.3 & 3.836 & 0.012 & 2 & 2.554 & 1.2 & 1.443 & 0.469 & 2.838 & 1.351 \\
\hline 2.7 & 2.839 & 0.009 & 1.6 & 2.090 & 0.9 & 1.493 & 0.430 & 1.843 & 1.540 \\
\hline
\end{tabular}

الجدول (6) البيانات المختبرية و المحسوبة للمسقط المنحرف المائل بنسبة (200/1)

\begin{tabular}{|c|c||c|c|c|c|c|c|c|c|}
\hline $\begin{array}{c}\mathrm{H}_{\mathrm{w}} \\
(\mathrm{cm})\end{array}$ & $\begin{array}{c}\mathrm{Q}_{\mathrm{m}} \\
(\mathrm{l} / \mathrm{s})\end{array}$ & $\begin{array}{c}\mathrm{q} \\
\left(\mathrm{m}^{3} / \mathrm{s} . \mathrm{m}\right)\end{array}$ & $\begin{array}{c}\mathrm{h}_{\mathrm{o}} \\
(\mathrm{cm})\end{array}$ & $\begin{array}{c}\mathrm{h}_{\mathrm{c}} \\
(\mathrm{cm})\end{array}$ & $\begin{array}{c}\mathrm{h}_{\mathrm{e}}(\mathrm{cm}) \\
\mathrm{center}\end{array}$ & $\mathrm{F}_{\mathrm{o}}$ & $\mathrm{h}_{\mathrm{e}} / \mathrm{h}_{\mathrm{c}}$ & $\begin{array}{c}\mathrm{Q}_{\mathrm{cal}} \\
(\mathrm{l} / \mathrm{s})\end{array}$ & $\mathrm{C}_{\mathrm{d}}$ \\
\hline \hline 9.2 & 17.859 & 0.060 & 7 & 7.122 & 4.3 & 1.026 & 0.603 & 19.255 & 0.927 \\
\hline 8.2 & 15.028 & 0.050 & 6.5 & 6.347 & 4 & 0.965 & 0.630 & 17.275 & 0.869 \\
\hline \hline 6.8 & 11.349 & 0.038 & 5 & 5.264 & 3.2 & 1.080 & 0.607 & 12.361 & 0.918 \\
\hline 5.2 & 7.589 & 0.025 & 4.2 & 4.025 & 2.4 & 0.938 & 0.596 & 8.028 & 0.945 \\
\hline \hline 4.4 & 5.9069 & 0.020 & 3.4 & 3.406 & 1.9 & 1.002 & 0.557 & 5.655 & 1.044 \\
\hline 3.7 & 4.5549 & 0.015 & 2.8 & 2.864 & 1.65 & 1.034 & 0.576 & 4.576 & 0.995 \\
\hline 3.2 & 3.6636 & 0.012 & 2.5 & 2.477 & 1.3 & 0.986 & 0.524 & 3.200 & 1.144 \\
\hline 2.7 & 2.8394 & 0.009 & 2 & 2.090 & 1.2 & 1.068 & 0.574 & 2.838 & 1.003 \\
\hline
\end{tabular}

رسمت علاقة بين عمق الماء الحرج (h) وعمق الماء عند حافة المسقط (h) كما موضحة في الأثــكال (4و 5) حيث وجدت العلاقات النالية لكل حالة من حالات المسقط العمودي و المنحرف:

المعادلات (9,8,7) توضح العلاقات بين (he و he ) للمسقط العمودي الأفقي و المائل بنسبة (200/1) و (100/1)

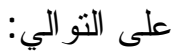

$\mathrm{h}_{\mathrm{e}}=0.676 \mathrm{hc}^{1.019}$

$\mathrm{h}_{\mathrm{e}}=0.617 \mathrm{hc}^{1.038}$ 
$\mathrm{h}_{\mathrm{e}}=0.525 \mathrm{hc}^{1.106}$

بينما المعادلات (12,11,10) توضح العلاقات بين (he و he ) للمسقط المنحرف الأفقي و المائل بنسبة (200/1) و

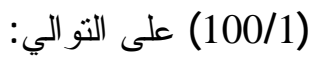

$\mathrm{h}_{\mathrm{e}}=0.546 \mathrm{hc}^{1.088}$

$\mathrm{h}_{\mathrm{e}}=0.509 \mathrm{hc}^{1.1}$

$\mathrm{h}_{\mathrm{e}}=0.383 \mathrm{hc}^{1.233}$

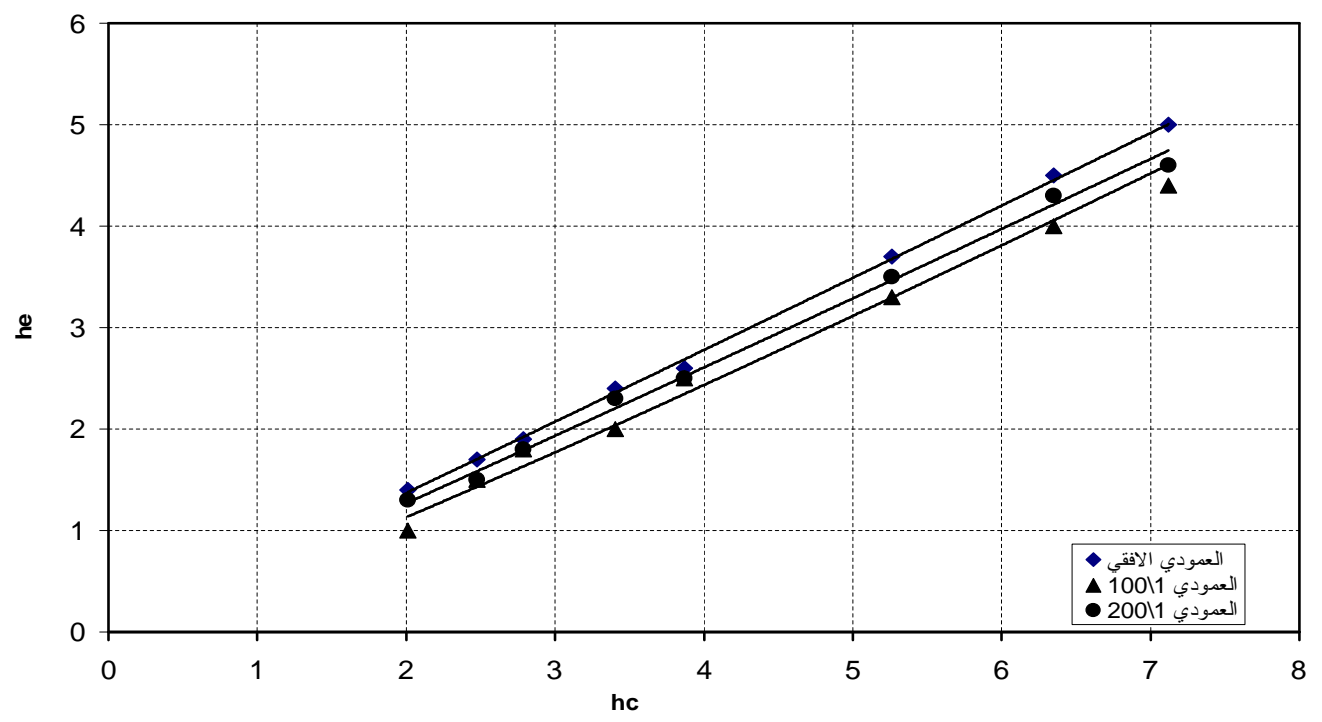

الثكل(4) علاقة عمق الماء الحرج (h) و عمق الماء عند حافة المسقط العمودي (he)

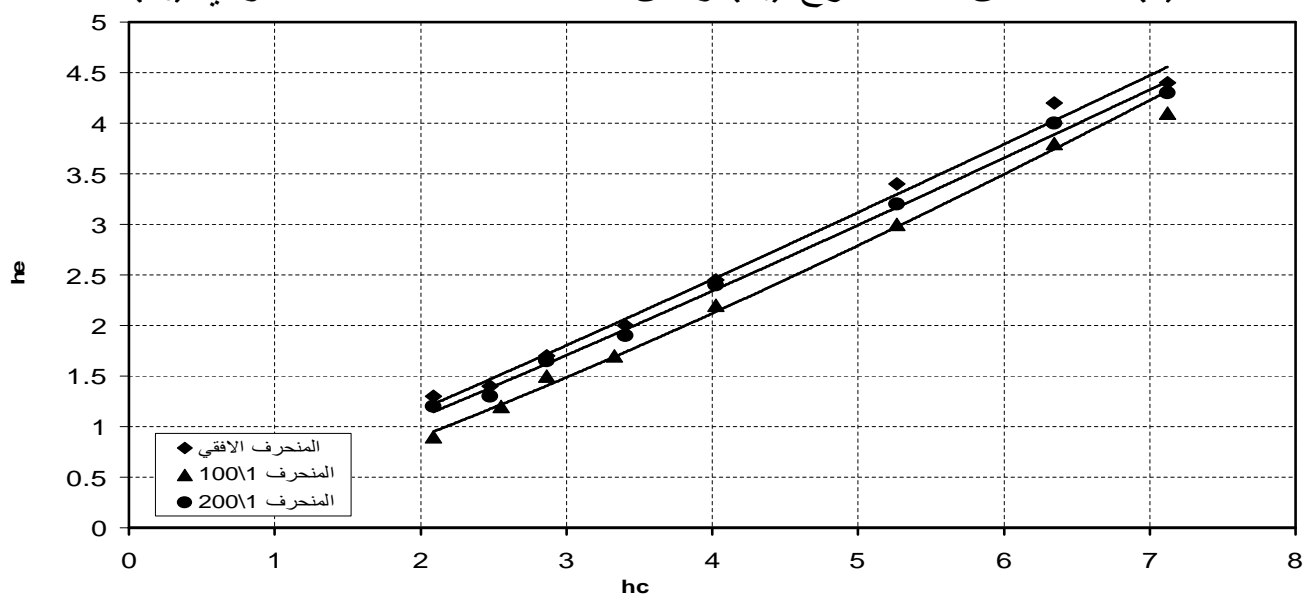

الثكل(5):علاقة عمق الماء عند حافة المسقط (he) و التصريف المحسوب (Qcal) للمسقط المنحرف

الأشكال (6و 7) يمثلان علاقة عمق الماء فوق حافة المسقط (h) و التصريف المحسوب (Q) للمسقط المنحرف و العمودي على التو الي.

من هذين الشكلين يلاحظ زيادة مقدار التصريف للنموذجين العمودي و المنحرف لنفس قيم(he)مع زيادة ميل القنــاة

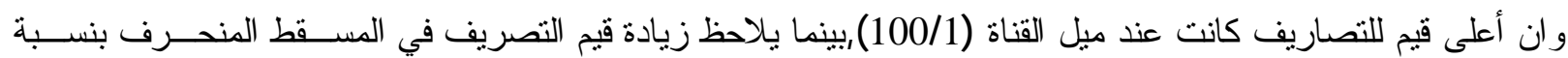
تصل الى (21\%) ثقريبا مقارنة بالمسقط العمودي عندما يكون ميل القناة (200/1). 


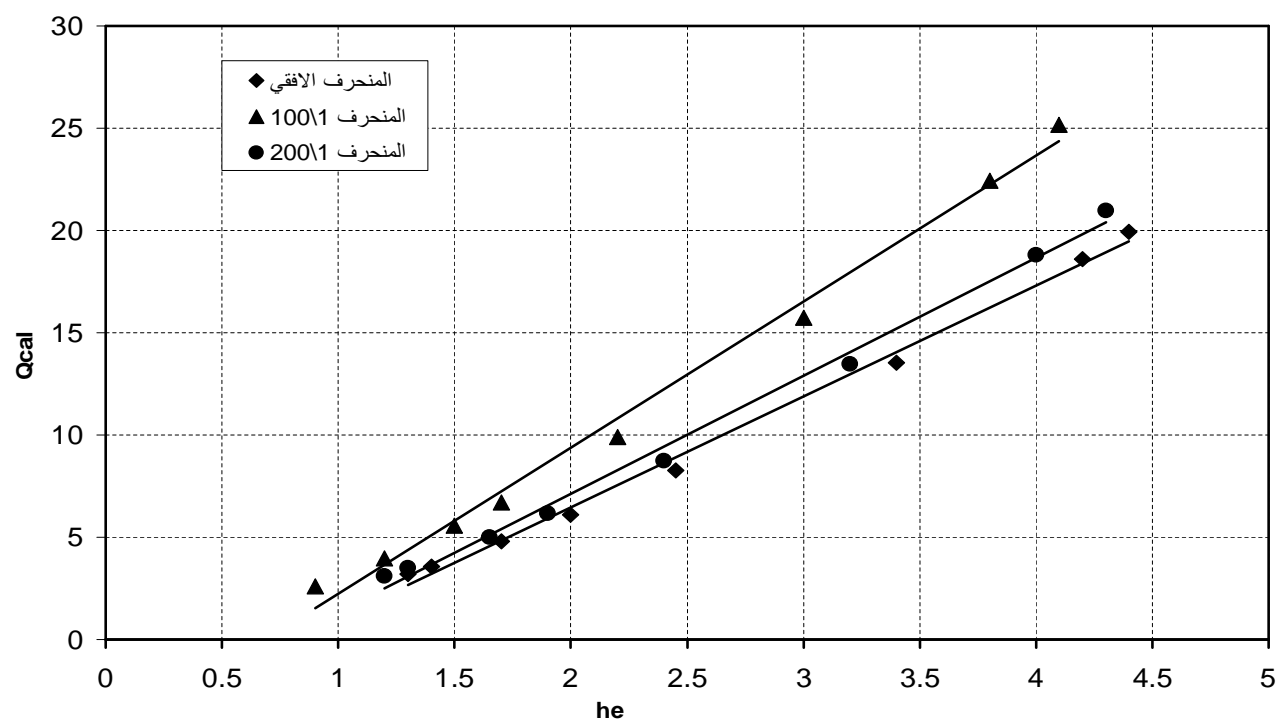

الشكل(6) علاقة عمق الماء عند حافة المسقط (he) و التصريف المحسوب (Qcal ) للمسقط المنحرف

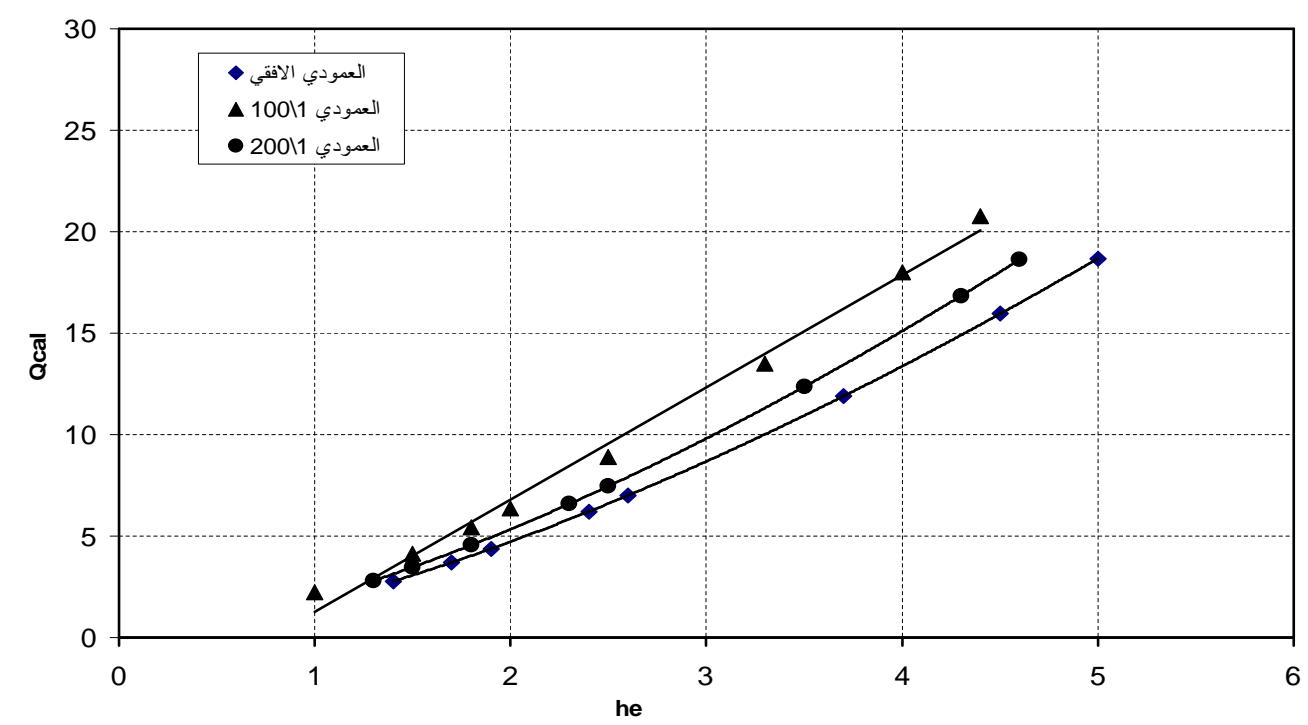

الثكل(7) علاقة عمق الماء عند حافة المسقط (he) و التصريف المحسوب (Qcal) للمسقط العمودي

الشكل (8) يمثل مقارنة قيم التصريف الحقيقي المقاس (Q) و المحسوب (Q) للمسقط المنحرف, حيث يلاحظ أن نسبة الخطأ لاتتجاوز (15\%) بين التصريف المقاس و المحسوب من جهة وتقاربها للمسقط الأفقي و المائل (200/1) من جهة أخرى.أما للمسقط المنحرف بميل (100/1) فيلاحظ تباعد قيم التصاريف المحسوبة عن المقاسة بشكل اكبر عن

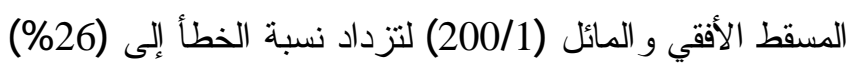
الشكل (9) يمنل مقارنة قيم التصريف الحقيقي (Qm ) و المحسوب (Q) للمسقط العمودي،حيث يلاحظ نقارب قيم التصريف المقاس و المحسوب لجميع الحالات للمسقط العمودي الأفقي و المائل (200/1) و (100/1) على التو الي بنسـبـة خطأ لا تتجاوز (12\%). 
محمد : تأثير ميل القتاة على خصائص الجريان للمسقط المائي العمودي والمنحرف

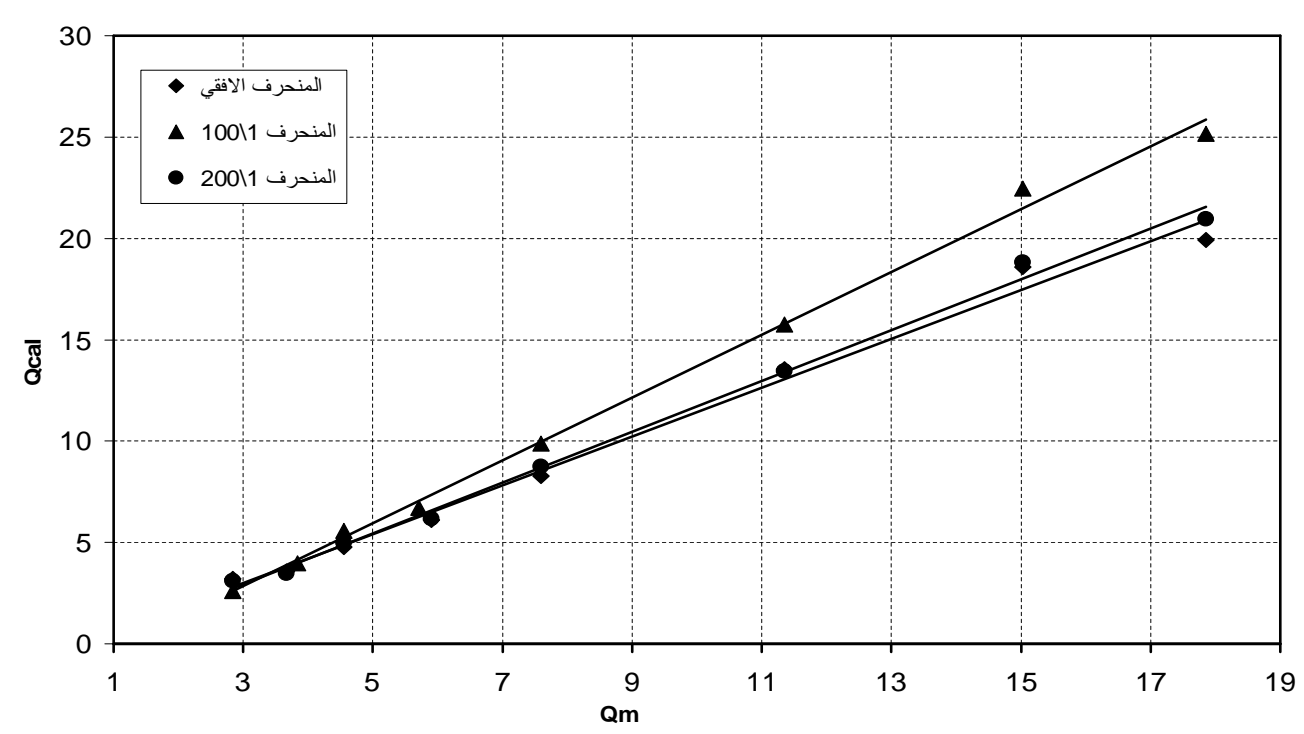

الثكل (8): علاقة التصريف الحقيقي (الدقاس) (Qm) و المحسوب (Q) للمسقط المنحرف

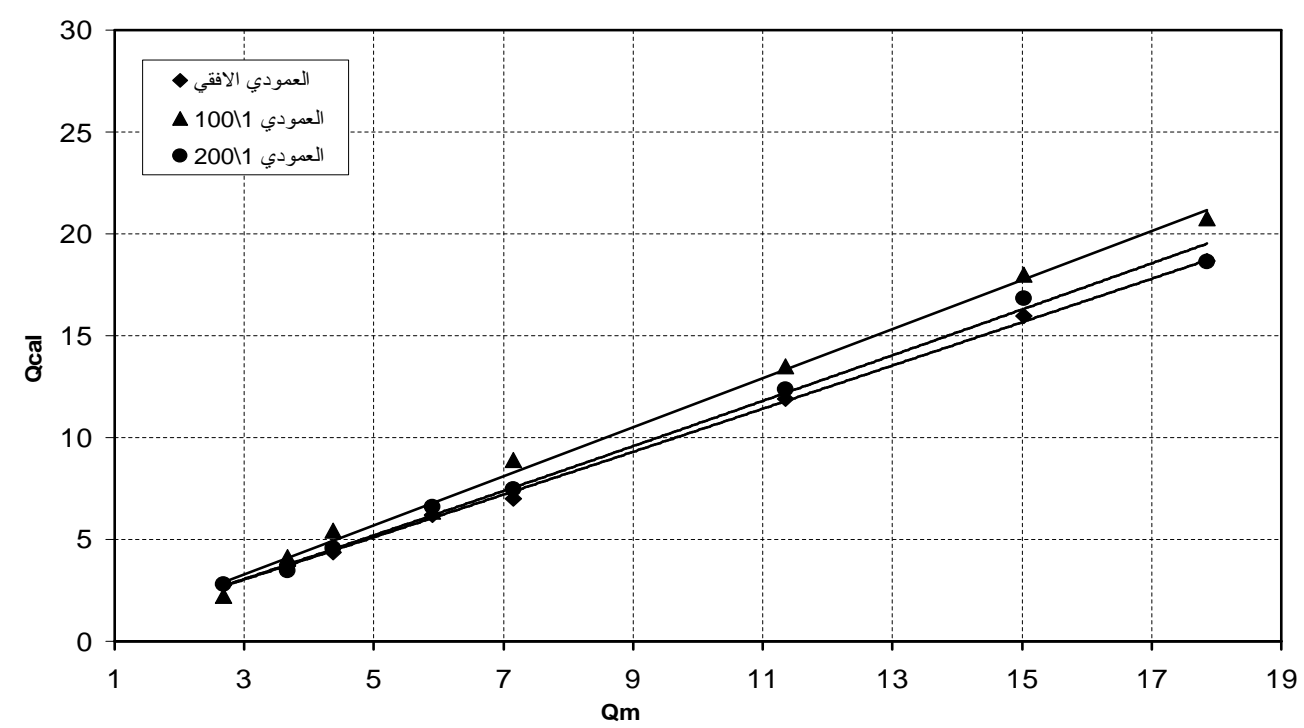

الثكل (9): علاقة التصريف الحقيقي (المقاس) (Qm) و المحسوب (Qcal) للمسقط العمودي

تم التوصل إلى المعادلات $(14,13)$ و التي نم إيجادها رياضيا باعتماد التحليل النظري باستخدام برنــامج التحليـلـل

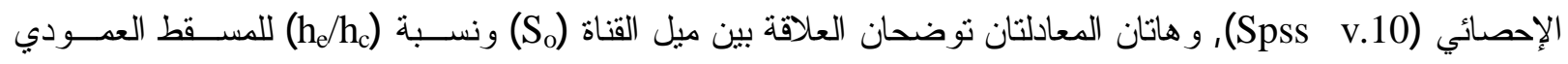
و المنحرف على التو الي ولجميع حالات الميل:

$h_{e} / h_{c}=-8.253 S_{o}^{2}-8.637 S_{o}+0.694$

$\mathrm{h}_{\mathrm{e}} / \mathrm{h}_{\mathrm{c}}=-453.23 \mathrm{~S}_{\mathrm{o}}^{2}-4.337 \mathrm{~S}_{\mathrm{o}}+0.616$

حيث أن: So

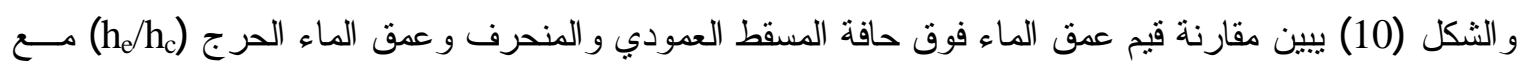

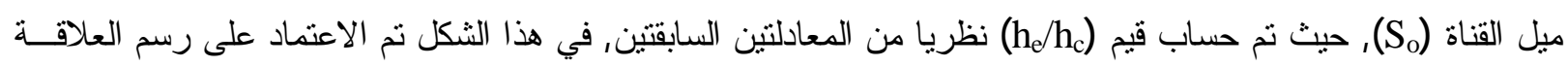

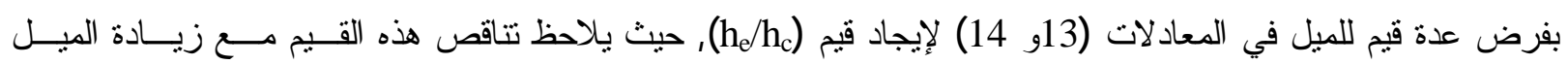


(Davis et. al., 1998), فكانت أنى قيمة لنسبة (h/h/h) بحدود (0.52) عند المسقط المنحرف و المائل (100/1), بينما

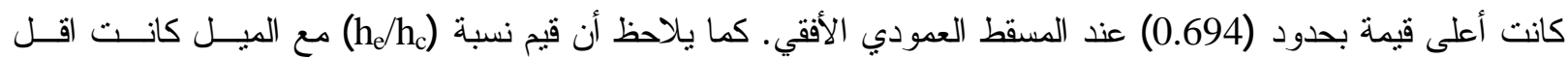
لجميع حالات المسقط المنحرف مقارنة بالمسقط العمودي.

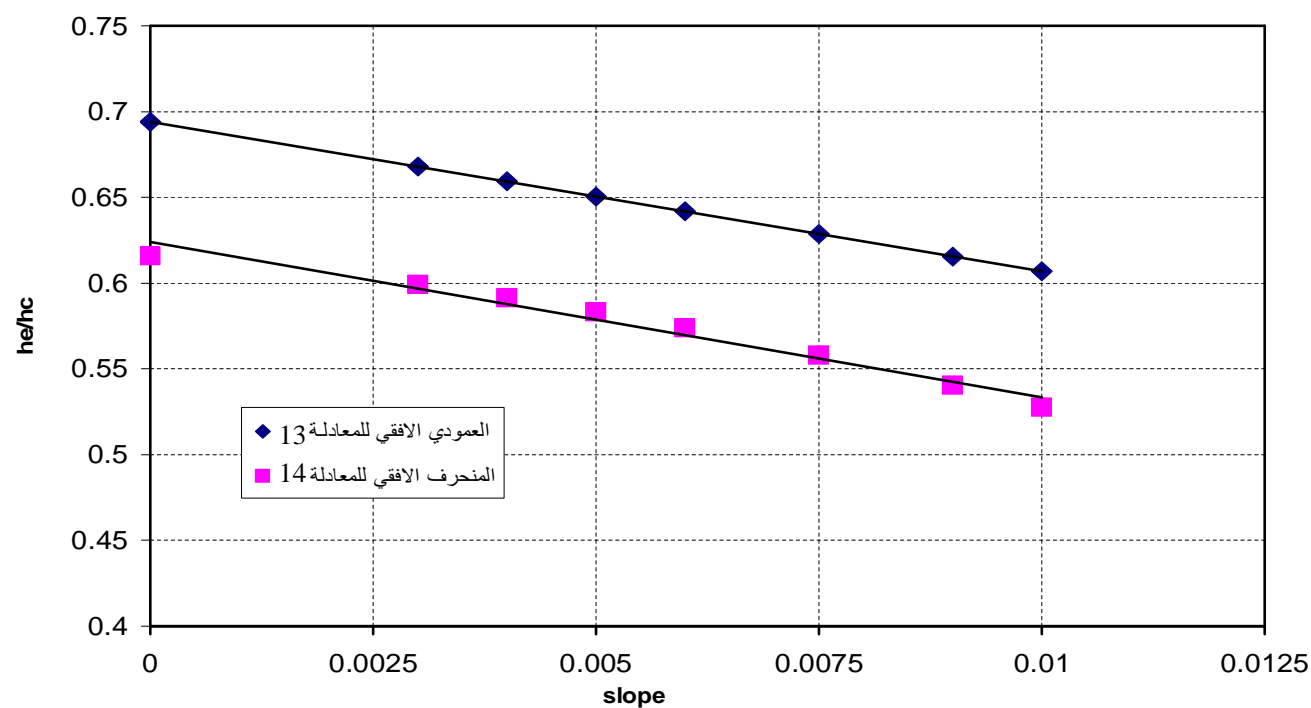

الثكل (10) علاقة ميل القناة ونسبة (h/he للمسقط العمودي و المنحرف للمعادلات (13 و 14)

الثكل (11) يمتل علاقة معامل التصريف (Che

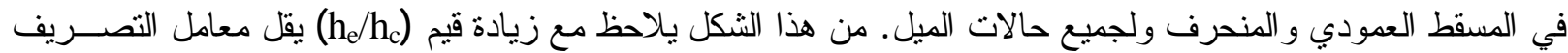

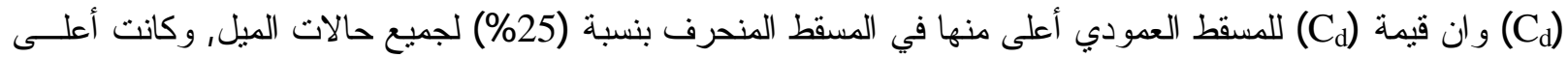

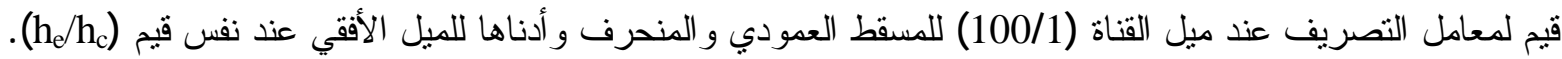

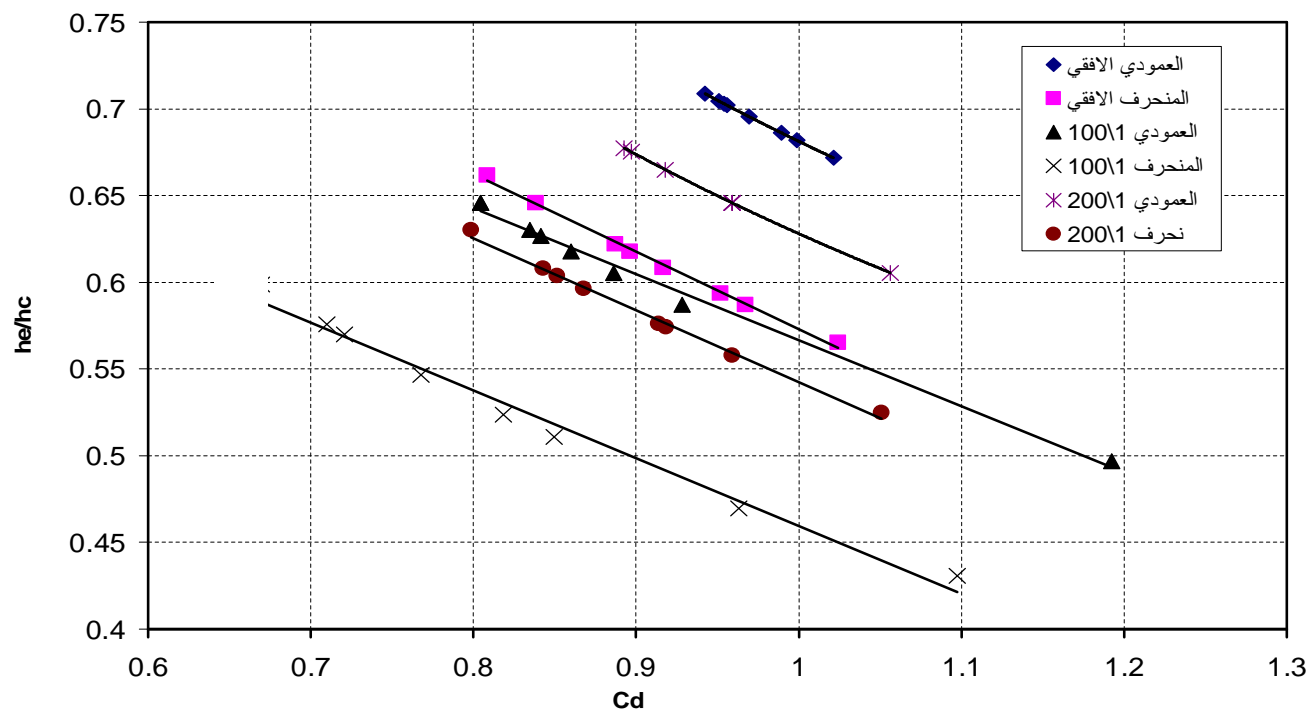

الثكل (11): علاقة معامل التصريف (C) مع نسبة (he/he للمسقط العمودي و المنحرف 
قدم هذا البحث در اسة وتحليل لتأثير ميل القناة على المسقط المائي كما قدم مقارنة بين المسقط العمودي و المنحرف ألماف

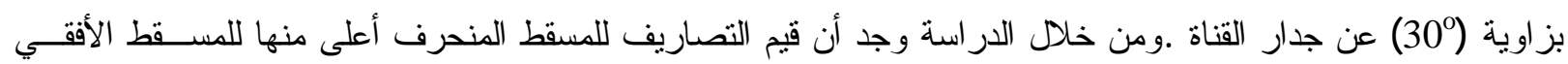

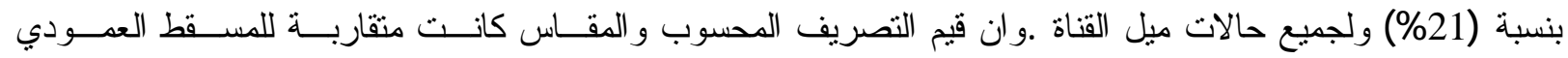
و المنحرف ولجميع حالات الميل بنسبة خطأ لا تتجاوز (15\%) للمسقط المنحرف و(12\%) للمسقط العمودي عــــا حالـــة المسقط المنحرف و المائل بنسبة (100/1) فقد بلغت نسبة الخطأ بين التصريف المقاس و المحسوب بنسبة (26\%), وبلغت أعلى نسبة زيادة في التصريف المحسوب (Qcal) في المسقط المنحرف و المائل (200/1) مقارنة بالمسقط العمودي حيـــث

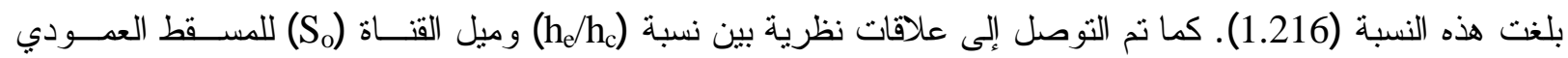
و المنحرف و علاقة نظرية لإيجاد النصريف المحسوب (Qcal) لجميع حالات المسقط العمودي و المنحرف, كمـــا أن قـيم معامل التصريف (Cdd للمسقط العمودي كانت أعلى من المنحرف بنسبة (25\%). عليه يمكن زيادة ميل القناة و اســتخدام مسقط منحرف لزيادة التصريف في القنو ات المفتوحة.

\section{(المصادر:}

Ahmed Y. M., Moayed S. K., Mwafaq Y. M., (2007). " Variation of Water Depth on Normal and Skewed Broad Crested Weirs", Tikret Univ. Jou. Of Eng., in press.

Bauer S. W. and Graf W. H. (1971). "Free overfall as flow measuring device", Jou. Of Irr. \& Dra. Eng., ASCE, 97(1), 73-83.

Davis A.C., Brian G.S. and Jacob R. P. (1998). "Flow Measurement in sloping channels with rectangular free overfall", Jou. Of Hyd. Eng., ASCE, 124(7), 760-763.

Ferro V. (1999). "Theoretical end-depth-discharge relationship for free overfall ", Jou. Of Irr. \& Dra. Eng., ASCE, 125(1),40-44.

Marchi E. (1993). " On the Free overfall ", Jou. Of Hyd. Res. Delft, The Netherlands, 31(6).

Mohapatra P. K., Bhallamudi S. M. and Eswaran V. (2001). " Numerical study of flow with multipl Free surfaces ", Int. Jou. Of Num. Methods Fluids, 36, 165-184.

Rajaratnam N., Durfakula M. and Spyridon B. (1976). " Roughness effects on rectangular free overfall", Jou. Of Hyd. Div., ASCE, 102(5), 599-614.

$$
\text { الطائي, احمد يونس, (2002) " در اسة هيدروليكية لأداء البو ابات العمودية و المائلة و المركبة على سد غاطس " أطروحة }
$$

تم اجراء البحث في كلية الهنسسة - جامعة الموصل 